\title{
Activation of Rabbit Platelets by Platelet-Activating Factor Derived from IgE-Sensitized Basophils
}

\author{
CHARACTERISTICS OF THE AGGREGATION AND ITS DISSOCIATION FROM \\ SECRETION
}

Peter M. Henson

From the Department of Immunopathology, Scripps Clinic and Research Foundation, La Jolla, California 92037

\begin{abstract}
A B S T RACT Platelet-activating factor (PAF) is liberated from antigen-stimulated, IgE-sensitized rabbit basophils and induces aggregation of platelets and secretion of their content of vasoactive amines. Experiments were performed to determine the relationship between these two platelet responses to this stimulus.
\end{abstract}

(a) Aggregation was initiated by appreciably lower concentrations of PAF than were required to produce even minimal release of constituents. PAF-induced aggregation was also resistant to agents which destroy ADP, such as creatine phosphate/creatine phosphokinase or apyrase, and occurred with platelets made unresponsive (refractory) to ADP. It was concluded that PAF can induce aggregation by a mechanism which is distinguishable from the release reaction and from the aggregating effect of ADP.

(b) Secretion can probably occur independently of aggregation because incubation without agitation resulted in secretion without detectable aggregation.

(c) Despite the suggestion that the two platelet responses can be independent, they may be induced by the same stimulus. This was indicated by experiments in which platelets specifically desensitized to PAF-induced secretion were also found to be unresponsive to PAF-stimulated aggregation. Moreover, identical levels of inhibition and identical inhibition profiles were obtained for both responses with serine esterase inhibitor, diisopropylphosphofluoridate, and with amino acid esters. It was concluded that the same stimulus-specific activable protease (esterase) was most likely involved in PAF-induced aggregation and secretion.

Received for publication 9 October 1975 and in revised form 15 April 1977.

The Journal of Clinical Investigation Volume 60 August 1977·481-490

\section{INTRODUCTION}

Platelet-activating factor $(\mathrm{PAF})^{1}$ is a soluble molecule which is secreted from IgE-sensitized rabbit basophils upon interaction with specific antigen. It is the active principle of a cooperative allergic reaction involving platelets and leukocytes first described by Barbaro and Zvaifler (1). The reaction was shown to involve IgE antibody (2), basophils (2-4), and the release from leukocytes of a soluble factor (4-6) termed platelet-activating factor (2). PAF has now been demonstrated in rats (7) and man (8-10) and appears to be a low molecular-weight lipid $(7,11)$ which binds to albumin (2). The material acts on platelets to stimulate aggregation (2) and secretion of vasoactive amines (5). In previous papers $(12,13)$, we defined the PAF-induced release reaction, described the generation of a specific state of desensitization in the platelets, and indicated the involvement of serine proteases, the cyclic AMP system, and intracellular contractile elements in the secretory event.

Recent experiments (14) have shown that PAFinduced release and aggregation occur in vivo as well as in vitro. In fact, a pathogenetic role for this mediator has been suggested in the deposition of immune complexes in acute immune complex disease (15), in the accumulation of platelets in immunologic skin lesions (16), and more recently, in IgE anaphylaxis $^{2}(14,17,18)$.

\footnotetext{
${ }^{1}$ Abbreviations used in this paper: ALMe, acetyl lysine methyl ester; BAMe, benzoyl-l-arginine methyl ester; DFP, diisopropylphosphofluoridate; EGTA, ethylene glycol-bis ( $\beta$-aminoethyl ether) $N, N, N^{\prime}, N^{\prime}$-tetraacetate; LMe, lysine methyl ester; PAF, platelet-activating factor; TAMe, tosyl-larginine methyl ester.

${ }^{2}$ Henson, P. M., and R. N. Pinckard. Manuscripts submitted for publication.
} 
Aggregation and release of constituents have been suggested by Holmsen (19) to represent different stages in a continuum of the platelet response to a stimulus. Agents which destroy or consume ADP have been used by a number of investigators to show that the aggregation induced by many stimuli, including collagen, or even thrombin, may result in part from the release of platelet ADP, which then causes the aggregation (20-23). In addition, the recent discovery of potent pharmacologic intermediates of the prostaglandin synthesis pathway, has suggested that these may represent the common platelet-activating material and that many stimuli activate by initiating prostaglandin synthesis (24-26).

Accordingly, we considered it important to examine the interdependence of the aggregation and release reaction induced in rabbit platelets by basophilderived PAF. The question may be posed in three ways. ( $a$ ) Is aggregation a consequence of the release, for example, of the aggregating-agent ADP? (b) Is aggregation required for the release reaction? (c) Are the two phenomena independent of each other, and if so, are they induced by the same activator? The experiments described herein are designed to address these queries.

\section{METHODS}

Animals. New Zealand white rabbits of either sex weighing from 2.5 to $4 \mathrm{~kg}$ were employed, as the source of platelets and PAF. Blood for platelets was not removed at intervals of $<1 \mathrm{mo}$.

Immunization. Horse radish peroxidase (type II, Sigma Chemical Co., St. Louis, Mo.) in saline was used as antigen. The initial injection comprised $10 \mathrm{mg}$ in saline subcutaneously into $2.5-\mathrm{kg}$ rabbits. Thereafter, animals were reimmunized with $5 \mathrm{mg} /$ injection at variable intervals (1$6 \mathrm{mo}$ ). The rabbits were bled for PAF production 7-10 days after each immunization.

Preparation of PAF. Blood was obtained from the central ear artery into $1 / 6 \mathrm{vol}$ of acid citrate dextrose (27). The procedure thereafter was similar to that described previously (2). The blood was sedimented at $500 \mathrm{~g}$ for $20 \mathrm{~min}$ at room temperature. The platelet-rich plasma was removed. The buffy coat layer on top of the erythrocytes was harvested with a siliconized (Siliclad, Clay Adams, Div. of Becton, Dickinson \& Co., Parsippany, N. J.) Pasteur pipette with tip bent at right angles. An equal volume of $2.5 \%$ gelatin in isotonic phosphate-buffered saline was added, and the mixture was incubated at $37^{\circ} \mathrm{C}$ for $30 \mathrm{~min}$ to sediment most of the erythrocytes. The supernatant cell suspension was removed and centrifuged at $500 \mathrm{~g}$ for $15 \mathrm{~min}$ at room temperature. The cell pellet was resuspended in a small volume of Tris-buffered Tyrode's solution with no $\mathrm{Ca}^{++}$or $\mathrm{Mg}^{++}$and with ethylene glycol-bis ( $\beta$-aminoethyl ether)$N, N, N^{\prime}, N^{\prime}$-tetraacetate (EGTA). Remaining erythrocytes were removed by hypotonic lysis. For each $2 \mathrm{ml}$ of cell suspension, $6 \mathrm{ml}$ of distilled water was added, and after $30 \mathrm{~s}, 2 \mathrm{ml}$ of $3.5 \%$ saline was used to restore isotonicity. Platelets were removed by four to five washes $(8 \mathrm{~min}$ at $180 \mathrm{~g}$ in the cold) in the 50 -ml plastic tubes using Tris-buffered Tyrode's solution without $\mathrm{Ca}^{++}$or $\mathrm{Mg}^{++}$. Cells were resus- pended to $1 \times 10^{7}$ leukocytes $/ \mathrm{ml}$ in Tris-Tyrode's with $0.25 \%$ bovine serum albumin. The cell concentration is important because if it is higher, the PAF apparently binds to the other cells present, and the yield is lowered. The albumin is essential and provides a carrier for the PAF (2). Antigen $\left(50 \mu \mathrm{g}\right.$ peroxidase for each $10^{7}$ cells) was added and incubated for $20 \mathrm{~min}$ at $37^{\circ} \mathrm{C}$. The cells were removed $(500 \mathrm{~g}$, for $10 \mathrm{~min}$ ), and the supernatant fluid containing PAF-albumin was tested, dialyzed against phosphate-buffered saline or Tris-buffered saline overnight at $4^{\circ} \mathrm{C}$, and frozen in aliquots at $-70^{\circ} \mathrm{C}$.

Extraction of PAF from albumin. PAF-albumin was added to a column of SM2 beads (Bio-Rad Laboratories, Richmond, Calif.) at room temperature, incubated $30 \mathrm{~min}$, drained, and washed with 1-2 vol Tyrode's solution. The hydrophobic beads extract the PAF from the albumin, and the effluent (containing the albumin) was shown not to activate platelets. The PAF was extracted from the beads with 3 vol $95 \%$ ethanol, spun at $12,000 \mathrm{~g}$ to remove any residual bovine serum albumin, and was stored in ethanol at $-70^{\circ} \mathrm{C}$. Aliquots were dried under vacuum and were then redissolved in Tyrode's solution with gelatin immediately before use.

Buffers. (a) Tyrode's solution: $\mathrm{NaCl}, 8 \mathrm{~g} / \mathrm{liter} ; \mathrm{KCl}$, $0.195 \mathrm{~g} /$ liter; $\mathrm{NaHCO}_{3}, 1.02 \mathrm{~g} /$ liter; $\mathrm{MgCl}_{2} 6 \mathrm{H}_{2} \mathrm{O}, 0.213 \mathrm{~g}$ /liter; $\mathrm{CaCl}_{2}$ (anhydrous), $0.145 \mathrm{~g} /$ /iter; glucose, 1 g/liter, $\mathrm{pH} 7.2$. (b) Tris-Tyrode's solution: same as above with $0.01 \mathrm{M}$ Tris (hydroxymethyl) amino-methane, instead of $\mathrm{NaHCO}_{3}$. (c) Tyrode's-gelatin: same as $a$, with $0.25 \%$ gelatin. $(d)$ Tyrode'sbovine serum albumin: same as $a$, with $0.25 \%$ bovine serum albumin. (e) Tyrode's-gelatin without $\mathrm{Ca}^{++}$, basic Tyrode's-gelatin with calcium omitted and a pH of 6.5. (f) Tyrode's-gelatin without $\mathrm{Ca}^{++}$and with EGTA; same as $e$ but with $0.1 \mathrm{mM}$ ethylene glycol tetraacetate. (g) TrisTyrode's without $\mathrm{Ca}^{++}$or $\mathrm{Mg}^{++}$.

Preparation of platelets. Rabbit platelets were prepared as described previously (28). Blood was taken into acid citrate dextrose (as for PAF production) and centrifuged at $500 \mathrm{~g}$ for $20 \mathrm{~min}$. The entire preparation procedure was carried out at room temperature and with plastic containers and pipettes. The platelet-rich plasma was removed and incubated at $37^{\circ} \mathrm{C}$ with $0.6 \mu \mathrm{Ci}\left[{ }^{3} \mathrm{H}\right]$ serotonin, binoxalate per $\mathrm{ml}$ (4.3 Ci/mmol, New England Nuclear, Boston, Mass.) for 15 $\mathrm{min}$. The platelets were sedimented at $1,800 \mathrm{~g}$ for $15 \mathrm{~min}$ and washed first in Tyrode's-gelatin without $\mathrm{Ca}^{++}$and with EGTA and then in Tyrode's-gelatin without $\mathrm{Ca}^{++}$. The platelets were resuspended in the latter buffer and diluted to 2.5 $\times 10^{9} / \mathrm{ml}$. For experiments they were diluted $1 / 10$ in Tyrode'sgelatin containing calcium. The preparation procedure is modified from that of Ardlie et al. (29) and preserves the platelet $\mathrm{Mg}^{++}$. Such platelets are readily aggregated by ADP in micromolar concentrations and are still discoid in shape. These characteristics are both indicative of normality. The platelets were kept at room temperature and used within 3 or $4 \mathrm{~h}$ of preparation. While their reactivity to ADP declined somewhat over this time, that to PAF remained unaltered.

Platelet stimuli. Thrombin (bovine, Parke, Davis \& Co., Detroit, Mich.) was diluted in Tyrode's-gelatin. Epinephrine (Parke, Davis \& Co.) was also diluted in Tyrode's-gelatin, but only immediately before use. Collagen was prepared by homogenization and then sonification of bovine tendon collagen (Sigma Chemical Co.) in saline. Alternatively, it was purchased from Worthington Biochemical Corp., Freehold, N. J. (soluble calf skin collagen) and diluted in Tyrode's solution before use. ADP was obtained from Sigma Chemical Co., and was freshly dissolved before use.

Release of serotonin. The standard reaction mixture contained $0.1 \mathrm{ml}$ of $2.5 \times 10^{y} / \mathrm{ml}$ platelets, varying amounts of 
PAF, and Tyrode's gelatin to $1 \mathrm{ml}$, yielding a final concentration of $2.5 \times 10^{8} / \mathrm{ml}$ platelets, previously found to be optimal for PAF (12). The platelets were generally added last. The reactions were performed in duplicate in $12 \times 75$ $\mathrm{mm}$ polystyrene tubes (Lancer, Sherwood Medical Industries, Inc., St. Louis, Mo.) at $37^{\circ} \mathrm{C}$ for $10 \mathrm{~min}$. Tubes were then cooled to $4^{\circ} \mathrm{C}$ in ice and centrifuged in the cold at $1,800 \mathrm{~g}$, $300 \mathrm{rpm}$ for $15 \mathrm{~min}$. A $0.1-\mathrm{ml}$ aliquot of the supernate was removed, and the radioactivity was counted in a mixture of equal parts of toluene and Aquasol (New England Nuclear, Pilot Chemicals Div., Watertown, Mass.) in a Beckman liquid scintillation counter (Beckman Instruments, Inc., Spinco Div., Palo Alto, Calif.). The secreted $\left[{ }^{3} \mathrm{H}\right]$ serotonin was usually expressed as a percentage of the total within the platelets, determined by lysis with Triton X-100 (Rohm \& Haas Co., Philadelphia, Pa.). Generally, $0.1 \mathrm{ml}$ of 2.5 $\times 10^{8} / \mathrm{ml}$ platelets contained $18-25 \times 10^{3} \mathrm{cpm}$. Background secretion from platelets incubated without stimulus was subtracted and never exceeded 5\% of the total. In some experiments, platelet suspensions were removed from the aggregation cuvette, immediately diluted $1: 5$ in ice-cold saline containing $5 \mathrm{mM}$ EDTA, and centrifuged as above, or centrifuged in a Brinkmann 3200 centrifuge (Brinkmann Instruments, Inc., Westbury, N. Y.) at $12,000 \mathrm{~g}$ for $30 \mathrm{~s}$ to immediately separate the supernate.

Release of nucleotides and enzymes. $100 \mu \mathrm{l}$ of ethanolEDTA extracts of supernates and platelet pellets was prepared for assay of ATP and ADP as recommended by Holmsen et al. (30). Light emission following addition of $500 \mu \mathrm{g}$ firefly lantern extract in $100 \mu \mathrm{l}$ water was measured in a Packard Tri-Carb liquid scintillation spectrometer (Packard Instrument Co., Inc., Downers Grove, Ill.), as suggested by Stanley and Williams (31). Lactic dehydrogenase and $\beta$-glucuronidase were assayed as described previously (28).

Platelet aggregation. The standard reaction mixture contained $0.05 \mathrm{ml}$ of $2.5 \times 10 \%$ ml platelets, varying amounts of stimulus, and Tyrode's-gelatin (containing calcium) to $0.5 \mathrm{ml}$. The Tyrode's was warmed to $37^{\circ} \mathrm{C}$ in the aggregation cuvette, the platelets and a Teflon-coated magnetic stirring rod was added, and the mixture was stirred at $1,100 \mathrm{rpm}$, at $37^{\circ} \mathrm{C}$ in an aggregometer (Chrono-Log Corp., Havertown, $\mathrm{Pa}$.) for 2-3 $\mathrm{min}$. Upon addition of the stimulus, the increase in light transmission was traced on a chart recorder. Aggregation was quantitated as described in the Results section.

Inhibitors. Diisopropylphosphofluoridate (DFP) was purchased from Sigma Chemical Co. and was dissolved in Tyrode's at $50 \mathrm{mM}$ immediately before use. Amino acid esters came from Sigma Chemical Co., except for lysine methyl ester, which came from Cyclo Chemicals, Miami, Fla., and were also dissolved in Tyrode's solution. Apyrase was obtained from Sigma Chemical Co. and was dissolved in Tyrode's solution.

\section{RESULTS}

\section{PAF-induced aggregation}

Typical tracings of the aggregation of rabbit platelets, induced by PAF are depicted in Fig. 1. The dose-response relationship may readily be seen. The aggregation process appears similar to that induced by most platelet-aggregating agents, including ADP, on rabbit platelets. Five parameters of the reaction could be seen and, in some cases, measured: $(a)$ the characteristic platelet shape change occurred immediately without any lag period (in contrast to that in-

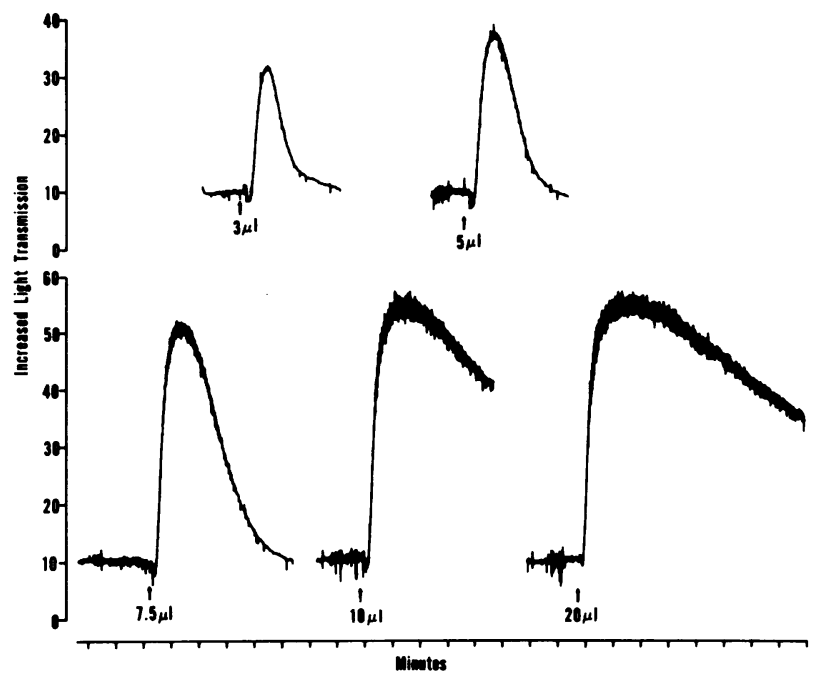

FIGURE 1 Tracing of PAF-induced aggregation. $0.5 \mathrm{ml}$ Tyrode's-gelatin containing $2.5 \times 10^{8} / \mathrm{ml}$ platelets was incubated at $37^{\circ} \mathrm{C}$ with stirring at $1,100 \mathrm{rpm}$. The initial shape change (decreased light transmission), followed by aggregation (increase in light transmission), produced in different cuvettes by increasing amounts (microliters) of extracted PAF (see Methods) is apparent.

duced by agents such as collagen); $(b)$ the rate of aggregation was linear for a considerable time; $(c)$ the extent of aggregation has been expressed as units of aggregation (i.e. divisions of 0.1 inch on the chart paper); the machine was calibrated so that maximum deflection (no platelets) represented about $80 \mathrm{U} ;(d)$ length of time aggregated; $(e)$ rate of disaggregation: aggregation induced by PAF was found to be transient in nature and was followed by a rapid disaggregation; this disaggregation occurred faster than with other stimuli (e.g. ADP and thrombin) used at concentrations to give similar degrees of aggregation.

The rate and extent of aggregation and the length of time aggregated were all directly proportional to the amount of PAF added. The rate of disaggregation was inversely proportional to concentration of PAFalbumin. This may be due to increased release of ADP, which itself could contribute to the aggregation. PAF is here expressed as microliters of a standard preparation of PAF-albumin. Extracted PAF induced identical patterns of aggregation. Since PAF has not yet been completely isolated, any quantitative differences between the effects of PAF bound to albumin compared with the extracted form cannot be determined. Qualitatively their effects were similar, and inasmuch as most experiments have been performed with both materials, the generic term PAF will be used throughout the text. Experiments were performed at $37^{\circ} \mathrm{C}$ and at platelet concentrations of 2.5 $\times 10^{8} / \mathrm{ml}$. 


\section{Is secretion required for aggregation?}

Dose response of stimuli. To investigate the relationship between PAF-induced aggregation and secretion, the effect of different concentrations of PAF on each of these parameters was examined (Fig. 2). It was found that $50 \%$ of the maximal release of serotonin (measured in the supernate from platelets undergoing aggregation) required about seven times as much PAF as $50 \%$ of the maximal aggregation (measured by the extent or rate of aggregation). This was true for both albumin-bound and extracted PAF. This provided the first suggestion that the aggregation response was not wholly a consequence of the release reaction. The data contrast with those obtained for thrombin in that $50 \%$ of the maximal secretion and aggregation was obtained with equivalent concentrations (data not shown). The dose-response relationship of PAF-induced secretion can be seen in Fig. 2. As described previously (12), maximum secretion often represents release of only $50-70 \%$ of the available serotonin.

PAF-induced secretion of nucleotides. The data in Fig. 2 do not rule out the possibility that the amount of PAF required to induce release of ADP (which would then stimulate aggregation) is less than that needed to liberate serotonin. Accordingly, the amount of ATP and ADP released from platelets by increasing doses of PAF was ascertained. Table I depicts the data. As described by others (23), ATP was the predominant nucleotide released. However, nearly maximal aggregation was seen at concentrations of PAF

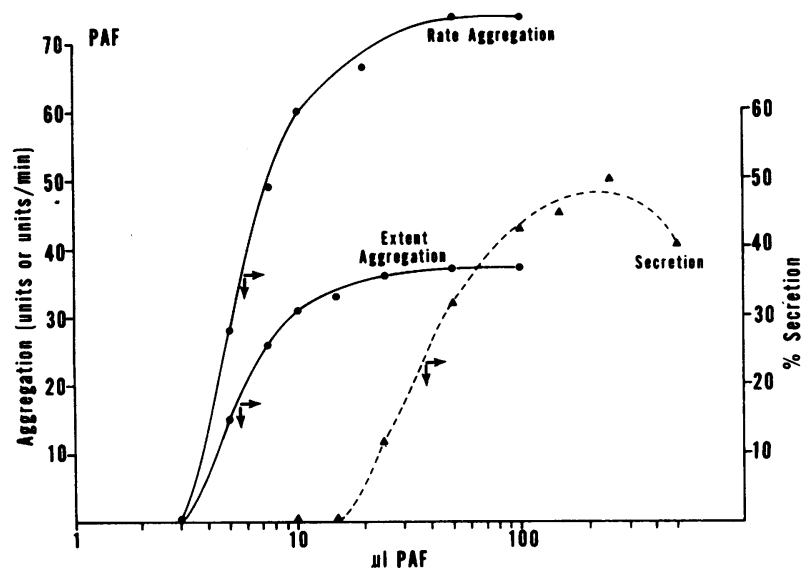

FIGURE 2 Comparison of aggregation and secretion of $\left[{ }^{3} \mathrm{H}\right]$ serotonin as a function of PAF concentration. Arrows indicate $50 \%$ maximal response. Similar results were obtained with PAF-albumin or with extracted PAF. The released $\left[{ }^{3} \mathrm{H}\right]$ serotonin was determined by centrifugation of the platelet suspension from the aggregation cuvette after the aggregation had been determined. Different methods were employed to prevent the repeated uptake of serotonin. Platelets were diluted in cold EDTA containing buffer before centrifugation. Alternatively, in later experiments, they were centrifuged rapidly at $12,000 \mathrm{~g}$. which induced minimal release of ADP, ATP, or serotonin. In these experiments, not enough detectable ADP was liberated or generated to have initiated the aggregation during the time period over which aggregation occurred (Table I).

The effect of creatine phosphate and creatine phosphokinase or of apyrase. To confirm the suggestion that ADP was not responsible for the PAF-induced aggregation, reagents which would consume or destroy any ADP which was released were employed (20-30). Fig. 3 shows the effect of creatine phosphate in the presence of creatine phosphokinase (22). All released ADP is converted to ATP. The reagents completely prevented ADP aggregation (although not the shape change). They also markedly diminished the aggregation caused by collagen. However, no effect was seen on PAF-induced aggregation. It is important to note that the concentrations of PAF used were chosen to be submaximal so that any possible effect of the creatine phosphate and creatine phosphokinase could be most readily determined. Similarly, (although not shown) apyrase, at concentrations 10 times those capable of completely preventing ADP aggregation, were unable to reduce that caused by PAF.

Enhancement of PAF-induced aggregation by ADP. Packham et al. (23) have shown synergistic effects between ADP and other platelet stimuli. To address this possibility in the case of PAF, experiments were performed in which PAF-induced aggregation was examined immediately after the addition of low doses of ADP. A typical experiment is shown in Fig. 4. In this experiment, ADP concentrations were chosen which were just below those required to give aggregation, although the shape change was still produced. Aggregation produced by low concentrations of PAF was enhanced by the ADP, though not to a great degree. If doses of ADP which just gave aggregation were used, the subsequent PAF response was again slightly enhanced, but not appreciably over the probable sum

TABLE I

PAF-Induced Aggregation and Release of Nucleotides and Serotonin

\begin{tabular}{ccccc}
\hline & & \multicolumn{3}{c}{ Release } \\
\cline { 3 - 5 } Volume PAF & Aggregation & {$\left[{ }^{3}\right.$ H $]$ Serotonin } & ATP & ADP \\
\hline$\mu l$ & \% of maximum & $c p m \times 10^{-3}$ & $n m o l$ & $n m o l$ \\
50 & 100 & 7.2 & $2.0 \pm 0.01$ & $0.3 \pm 0.07$ \\
25 & 100 & 7.7 & $1.4 \pm 0.02$ & $0.3 \pm 0.02$ \\
10 & 100 & 6.0 & $0.9 \pm 0.02$ & $0.3 \pm 0.08$ \\
5 & 100 & 2.7 & $0.7 \pm 0.01$ & $0.2 \pm 0.03$ \\
2 & 86 & 0.4 & $0.3 \pm 0.03$ & $0.1 \pm 0.03$ \\
0 & - & 0.3 & $0.3 \pm 0.01$ & $0.1 \pm 0.05$ \\
Total & & 10.6 & $6.8 \pm 0.40$ & $0.7 \pm 0.20$ \\
\hline
\end{tabular}




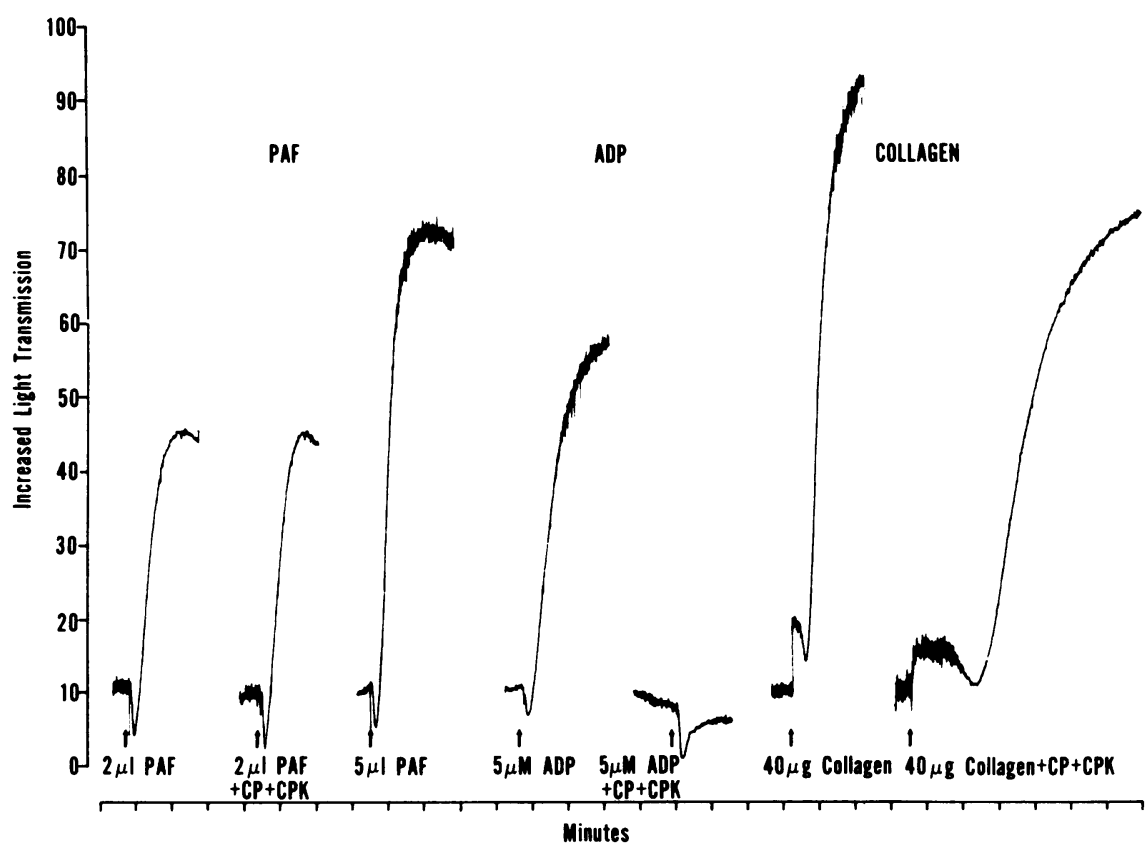

FIGURE 3 The effect of creatine phosphate (CP) and creatine phosphokinase (CPK) on aggregates of washed rabbit platelets by PAF. CP was used at $2 \mathrm{mM}$ final concentration and $\mathrm{CPK}$ at $2 \mathrm{U}$ per cuvette $(4 \mathrm{U} / \mathrm{ml})$ and were added $1 \mathrm{~min}$ before the stimulus. Inhibition of ADP and collagen-induced aggregation are depicted; inhibition of PAF-stimulated aggregation was not found. A potent PAF preparation was used at submaximal concentration in these experiments.

of the two reactions. Enhancement, as seen by Packham et al. (23), with collagen and thrombin, was not observed.

The effect of PAF on platelets made refractory to $A D P$. Prolonged incubation of rabbit platelets with ADP renders them refractory to additional ADPinduced aggregation. Accordingly, as depicted in Fig. 5 , millimolar concentrations of ADP were added to washed platelets in an aggregometer cuvette. At these high ADP concentrations, the shape change still occurred, but aggregation was minimal. Further addition of an aggregating dose of ADP $(5 \mu \mathrm{M})$ was without effect. However, PAF still induced normal aggregation, indicating again that this effect is probably not due to ADP. The normality of a PAF-induced, aggregation dose response using platelets rendered unresponsive to ADP as described above is also shown in Fig. 6, indicating that PAF induces aggregation in these circumstances even at low doses.

The effect of $A D P$ on platelets desensitized to $P A F$. To determine whether PAF aggregation would render platelets refractory to an ADP effect, ADP was added to platelets which had previously responded to PAF (aggregation and deaggregation). A normal effect of ADP was seen (Fig. 5).

We had previously described the induction of a state of specific desensitization in rabbit platelets to the induction of the release reaction by PAF (12).
Experiments were performed to determine whether such a desensitized state could be induced to PAFinduced aggregation and whether the desensitized platelets were still responsive to ADP. Accordingly,

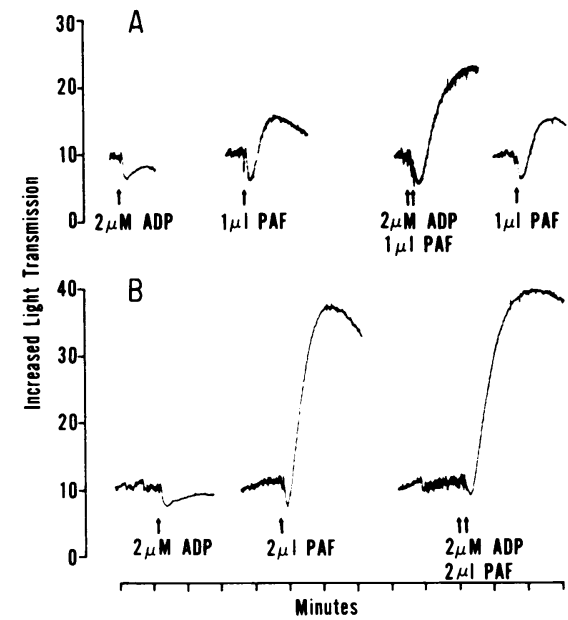

FIGURE 4 The effect of ADP on PAF-induced aggregation. The ADP concentration, which involved only a shape change and was still higher than the concentration detected in the medium after PAF had induced maximal platelet aggregation (Table I), was added simultaneously with low doses of PAF. $A$ and $B$ represent separate experiments with different preparations of platelets and of PAF. A, extracted PAF; B, PAFalbumin. 


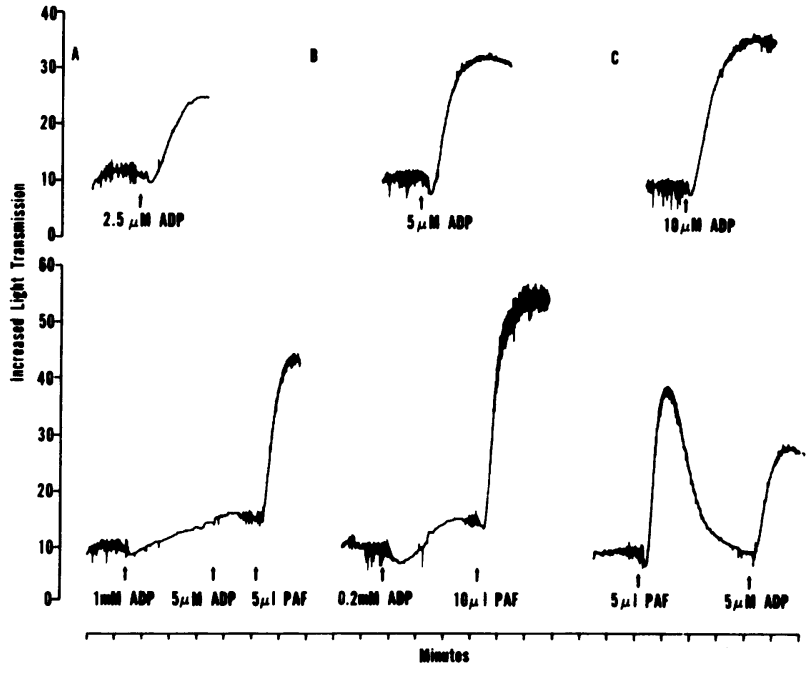

FIGURE 5 The effect of PAF on platelets made refractory to the aggregating action of ADP. The response of washed platelets from rabbits to ADP alone is depicted at the top. (A, B) A high concentration of ADP rendered the platelets unable to aggregate to a subsequent aggregating dose. However, they were still normally responsive to PAF. (C) Platelets which have responded to PAF still aggregated normally to ADP.

platelets were rendered unresponsive to PAF-induced release of serotonin. The control platelets (incubated with EGTA alone) or those rendered refractory to ADP aggregation, responded normally. Similarly the PAF-desensitized platelets could be induced to release serotonin by other stimuli such as collagen, thrombin, or C3b (not shown here, but reported in reference 12). Platelets desensitized in this manner were also

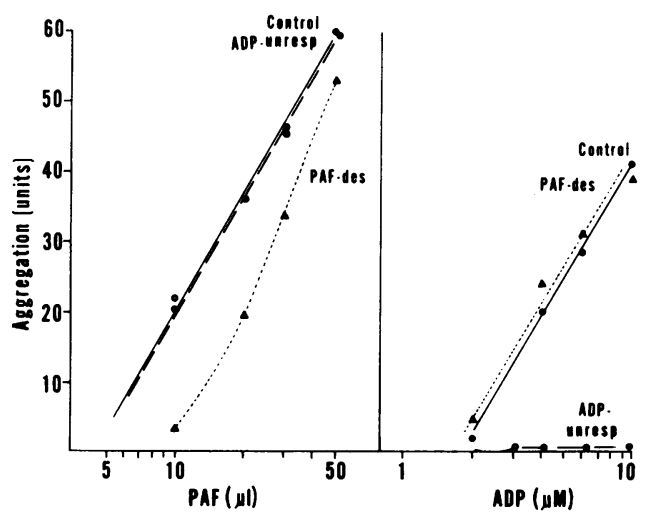

FIGURE 6 Aggregation of platelets rendered unresponsive (unresp) to ADP or desensitized (des) to PAF. 3-ml platelets $\left(2.5 \times 10^{\%} / \mathrm{ml}\right)$ were incubated with $5 \mathrm{mM}$ EGTA (control), EGTA and $1.5 \mathrm{ml}$ PAF (PAF-des), or EGTA and $100 \mathrm{mM}$ ADP (ADP-unresp) for $20 \mathrm{~min}$ at $37^{\circ} \mathrm{C}$. After incubation or, in the case of PAF desensitization, after incubation and washing, they were tested for aggregation induced by PAF or ADP. The ordinate represents units of aggregation (see Figs. 1 and 2). found to be less reactive to the aggregating action of PAF (Fig. 7). In contrast, they responded normally to increasing concentrations of ADP. The ability of PAF to induce a state of desensitization with regard to both the release reaction and aggregation, suggests a possible common step in the initiation of each process.

\section{Is aggregation required for secretion?}

This question is difficult to answer experimentally. PAF can be added to a platelet suspension in an aggregometer tube without a stirring bar in a manner which induces release without any detectable aggregation (Fig. 8). This release of serotonin (25.1) is less than that seen with stirring (35.6), but inasmuch as there is no mixing of PAF with the platelet suspension in the absence of stirring, this difference would be expected. By pouring platelet suspensions $(0.5 \mathrm{ml})$ into $2 \mathrm{ml}$ glutaraldehyde $(0.2 \%$ final concentration) followed by microscope examination, it was determined that $<5 \%$ of the platelets was adherent to each other at the point of sampling $(2 \mathrm{~min})$, whereas $25 \%$ of the serotonin had been released. With stirring, the release was increased to $35 \%$, but most of the platelets were seen to be aggregated. The data from this and three other experiments suggest strongly that strong platelet-platelet binding is not required for PAF-induced release of serotonin.

\section{Inhibition by DFP and amino acid esters}

We have previously postulated the requirement for activation of a DFP-inhibitable serine protease in PAFinduced secretion $(13,32)$. If the same PAF-receptor interaction can lead to both aggregation and secretion, DFP might be expected to inhibit both phenomena in a similar fashion. Data presented in Fig. 9 show this to be the case. $50 \%$ inhibition of both responses was obtained with $50 \mathrm{mM}$ DFP. It was further determined that pretreatment of platelets or PAF with the inhibitor (which acts on serine proteases

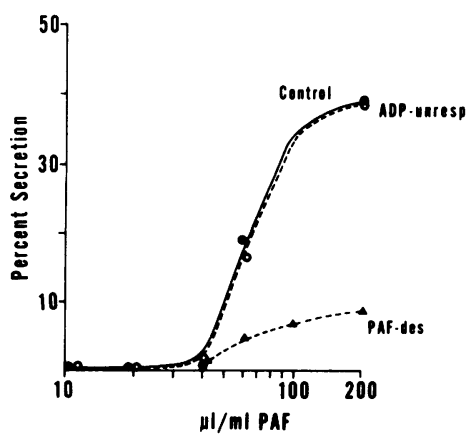

Figure 7 Secretion from platelets desensitized (des) to PAF or made unresponsive (unresp) to ADP. The platelets were identical to those depicted in Fig. 6. 

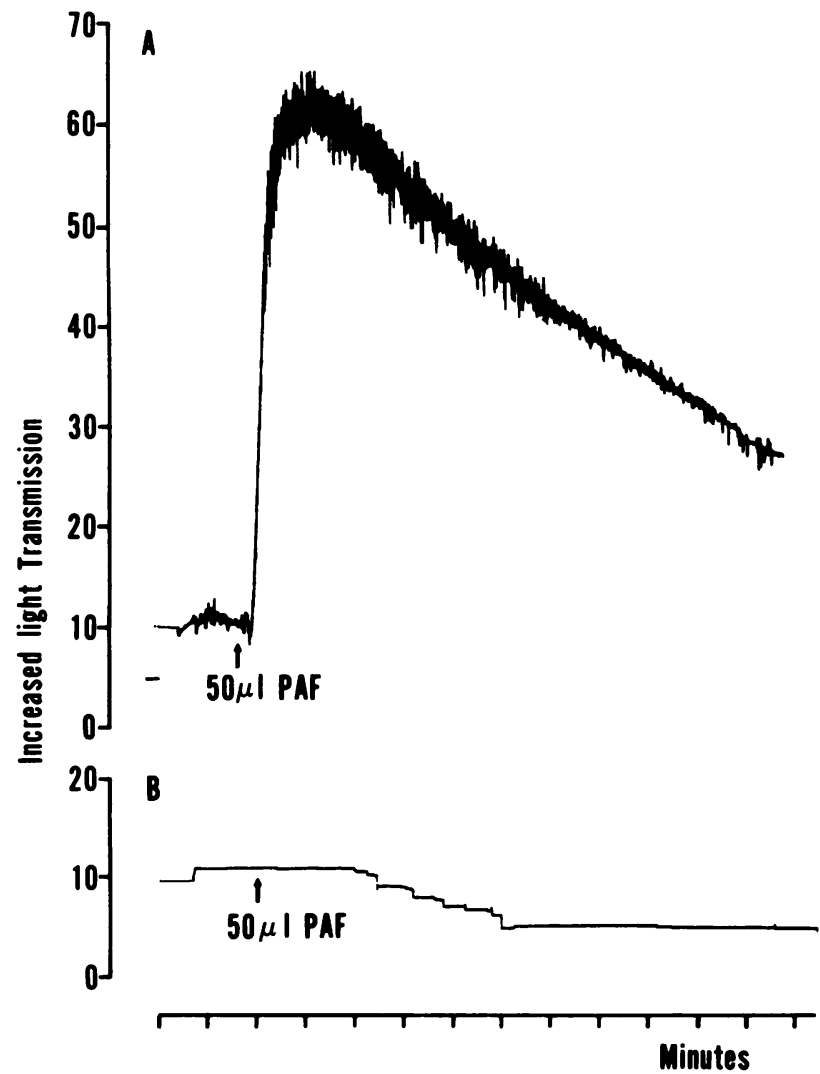

FIGURE 8 (A) $35.4 \%$ release of serotonin from platelets incubated with PAF under conditions in which they were stirred at $1,100 \mathrm{rpm}$; (B) $25.1 \%$ release of serotonin without stirring but in otherwise identical conditions.

in an irreversible manner) followed by washing or dialysis, did not prevent the effects of their subsequent interaction. The activation of a precursor serine protease(s) is suggested.

As previously reported (13), low molecular weight amino acid esters inhibit PAF-induced secretion, presumably by acting on the putative activable serine protease. The data presented in Table II show the identical specificity of inhibition and degree of inhibition of aggregation as of secretion. Lysine methyl ester $(\mathrm{LMe})$ is inhibitory, but the acetylated derivative (ALMe) is not. Similarly, tosyl-l-arginine methyl ester (TAMe) is inhibitory, but benzoyl-l-arginine methyl ester (BAMe) is not. These differences between $\mathrm{LMe}$ and ALMe or between TAMe and BAMe were not observed with collagen- or thrombin-induced platelet release reactions. ${ }^{3}$

\section{DISCUSSION}

PAF stimulates platelet secretion, i.e. the 'release reaction' of Grette (32) and also platelet aggregation.

\footnotetext{
${ }^{3}$ Henson, P. M., R. Landes, and E. L. Becker. Manuscript in preparation.
}

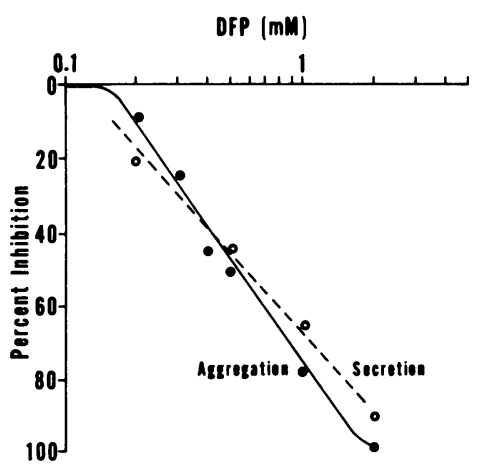

FIGURE 9 Inhibition of PAF-induced aggregation and secretion by DFP. DFP was included in the reaction mixture. However, when platelets or PAF were pretreated with DFP for $20 \mathrm{~min}$ at $27^{\circ} \mathrm{C}$ and washed or dialyzed, no inhibition was seen.

The data presented herein indicate that these are two separable responses of the platelet to PAF and are not necessarily interdependent.

$P A F-i n d u c e d$ aggregation. Incubation of PAF with platelets under conditions of stirring or agitation results in a rapid shape change and then aggregation in a manner similar to that seen with other platelet stimuli. The platelets remain aggregated, however, for only a short period and then undergo spontaneous disaggregation. The aggregation is temperature-dependent, requires calcium (2), and is inhibitable by DFP and certain amino-acid esters.

Experiments in which PAF extracted from the albumin (to which it is usually bound during preparation) were compared to albumin-bound PAF revealed no significant qualitative differences between the two species with regard to aggregation and release.

The characteristics of the aggregating effects of PAF are similar to those of PAF-induced release $(12,13)$. In addition, DFP was found to inhibit both aggregation and release at the same concentration and also in the same manner. Furthermore, an identical

\section{TABLE II}

Inhibition of PAF Aggregation by Amino Acid Esters

\begin{tabular}{lcc}
\hline & \multicolumn{2}{c}{$\mathrm{pl}_{\text {so }}$ PAF* } \\
\cline { 2 - 3 } & Aggregation & Secretion \\
\hline LMe & $3.3 \pm 0.07$ & $2.8 \pm 0.05$ \\
ALMe & $>1.5$ & $>1.0$ \\
TAMe & $3.6 \pm 0.1$ & $3.6 \pm 0.1$ \\
BAMe & $2.3 \pm 0.13$ & $2.0 \pm 0.08$ \\
\hline
\end{tabular}

Mean \pm SEM.

* PAF concentrations which stimulate approximately $50 \%$ of maximal aggregation or $35 \%$ secretion were employed.

$\ddagger \mathrm{pl}_{50}$ represents the negative logarithm of the concentration of ester to achieve $50 \%$ inhibition. 
pattern of amino-acid ester-induced inhibition was found for both aggregation and secretion. Thus, LMe was inhibitory, but ALMe was not. TAMe was inhibitory, but BAMe was not. Recent experiments (33) have suggested that different stimuli activate different proteases in rabbit platelets and this ester specificity suggests that the identical serine protease is involved in both PAF-induced activities. Inhibition only occurred if the DFP was present during the reaction, not if PAF or platelets were pretreated and then dialyzed or washed. This has been taken as circumstantial evidence for the requirement of a serine protease which is activated by the stimulus, or stimuluscell interaction, and is only then inhibitable by DFP $(13,33,34)$. It is suggested therefore, that aggregation as well as release involves activable serine proteases, and the data are consistent with the hypothesis that both phenomena may be responses to the same molecule (PAF) reacting with the same receptor. This has yet to be proven and will require purification of the active principle of the PAF preparations for verification. It should be noted, however, that the action of PAF noted here is not due to contaminating arachidonic acid, inasmuch as PAF effects are not inhibited by indomethacin or aspirin (35), and arachidonic acid works normally on PAF-desensitized platelets. ${ }^{4}$

Further evidence for both secretion and aggregation resulting from identical activation steps comes from the desensitization data. Platelets specifically desensitized to PAF release (12) were here shown to exhibit reduced responsiveness to the aggregating action of PAF, although they were normally responsive to ADP. In contrast, ADP-refractory platelets reacted normally to PAF. The greater effect of desensitization on secretion than on aggregation may reflect the requirement for higher PAF concentration for the former effect.

Aggregation does not require the release reaction. Evidence has been presented that release of ADP (or other material) from platelets stimulated by PAF is not the primary cause of the aggregation. This has been contrasted with the effects of collagen. (a) As described above, platelets which were completely refractory to the aggregation induced by ADP were normally responsive to PAF. (b) $\mathrm{Up}$ to seven times more PAF was required to induce release than to initiate aggregation, and almost maximal aggregation was seen with doses of PAF which did not induce release of serotonin. This difference was seen with albuminbound as well as with extracted PAF and was additionally evident when the release and aggregation were both performed in the same cuvette under conditions of stirring at $1,100 \mathrm{rpm}$. (c) The amount of ADP released from platelets by PAF at doses which

\footnotetext{
${ }^{4}$ Shaw, J. O., and P. M. Henson. Unpublished observation.
}

can induce aggregation is not enough to cause the aggregation. In addition, too little of the released ATP is in evidence for conversion to free and detectable ADP to account for the aggregation. In addition, because the responses of platelets to PAF are so fast, ADP generated from released ATP (the predominant nucleotide in rabbit platelets) may be too slow to provide enough ADP to greatly affect the PAF response. These two observations may explain why so little evidence of ADP contribution to the aggregation was found. $(d)$ Creatine phosphate and creatine phosphokinase or apyrase readily inhibited ADP-induced aggregation as expected. These reagents also inhibited aggregation induced by collagen. However, as shown in Fig. 3, no effect on the PAF-induced aggregation was detected. This observation strongly suggests that PAF aggregation can occur in the absence of ADP.

The possibility that low doses of ADP could sensitize the platelets to a synergistic action of PAF (23) was investigated, but a slight enhancement of PAF aggregation was seen only at the lower doses of PAF. The possibility that PAF could sensitize the platelets to a synergistic action of ADP, as has been reported for other stimuli (23) was also considered. However, PAF induced normal aggregation of platelets made refractory to ADP aggregation by high concentrations of ADP. Moreover, ADP did not induce an increased aggregation response when added after PAF had already stimulated aggregation (Fig. 5).

The experiments suggested strongly that PAFinduced aggregation is not solely a consequence of the release of ADP. It is also of interest that, in our work, PAF induces an aggregation of washed rabbit platelets which consistently is shorter-lived and disaggregates more readily than any other stimulus so far examined. The combined data suggest that the aggregation is not a consequence of the secretory process.

Aggregation is not required for secretion. The experiments also suggest that PAF induces the release reaction by a mechanism which is independent of the aggregation. Incubation of platelets with PAF in the complete absence of stirring or agitation eliminated aggregation but did not abolish secretion. Microscope examination of rapidly fixed platelet suspensions confirmed the lack of aggregation. The lower levels of release observed in the nonstirred situation almost certainly reflect the insufficient mixing inherent in this experimental design. Aggregation of platelets seems, therefore, not to be involved in PAFinduced secretion. While this is certainly true for firmly adhering aggregates of more than two platelets, the conclusion cannot be taken to its limit, because it cannot be shown that transient platelet-platelet interactions may not be involved.

Conclusions. The activation of rabbit platelets by $\mathrm{PAF}$, as with other stimuli, leads to rapid aggregation 
and secretion. It is suggested that the interaction of PAF with its platelet receptor directly or indirectly activates a unique precursor serine protease. This can then stimulate, in order, pathways leading to both aggregation and secretion (see also 12, 21,34). Since both require energy and $\mathrm{Ca}^{++}$and probably involve cyclic AMP and intracellular contractile elements $(12,34)$, some portions of these pathways may be identical. Nevertheless, neither platelet response is dependent upon the other, and in fact, it may be possible to activate one without the other. It is, of course, possible that both aggregation and secretion are manifestations of the same overall cell response to PAF stimulation, because in this system, as is also true with other stimuli, clear-cut evidence of complete inhibition of one without the other has not yet been obtained.

Holmsen (19) has proposed that shape changes, aggregation, secretion of serotonin and nucleotides, and finally secretion of lysosomal enzymes represent four sequential stages in an overall response of platelets to a stimulus. Weaker stimuli initiate only the earlier stages. This concept of similar pathways involving $\mathrm{Ca}^{++}$and contractile elements (actomyosin) for each type of platelet response is compatible with the information obtained for PAF $(12,13)$ and is similar to our hypothesized mechanistic similarity between responses to stimuli in a wide variety of cells, including platelets (34). However, there may be separable processes involved in aggregation and secretion which could, on the one hand, involve largely common intracellular pathways, diverging only in the final cell reaction, or, alternatively, largely independent pathways utilizing similar intracellular elements.

\section{ACKNOWLEDGMENTS}

The author is indebted to Tomi Dearmont for typing the manuscript and to Gerry Sandford and Gay Lee for preparing the illustrations, and to Dr. James Shaw for performing the nucleotide assays.

This investigation was supported by grants HL 16411-01, GMS 19322-04, and HL 17786-01 from the National Institutes of Health, U.S. Public Health Service.

\section{REFERENCES}

1. Barbaro, J. F., and N. J. Zvaifler. 1966. Antigen induced histamine release from platelets of rabbits producing homologous PCA antibody. Proc. Soc. Exp. Biol. Med. 122: $1245-1247$.

2. Benveniste, J., P. M. Henson, and C. G. Cochrane. 1972. Leukocyte-dependent histamine release from rabbit platelets. The role of IgE, basophils and a platelet-activating factor. J. Exp. Med. 136: 1356-1377.

3. Siraganian, R. P., and A. G. Osler. 1971. Destruction of rabbit platelets in the allergic response of sensitized leukocytes. II. Evidence for basophil involvement. J. Immunol. 106: 1252-1259.
4. Henson, P. M., and J. Benveniste. 1971. Antibodyleukocyte-platelet interactions. In Biochemistry of the Acute Allergic Reactions. K. F. Austen and E. L. Becker, editors. Blackwell Scientific Publications, Ltd., Oxford. 111-126.

5. Henson, P. M. 1970. Release of vasoactive amines from rabbit platelets induced by sensitized mononuclear leukocytes and antigen. J. Exp. Med. 131: 287-306.

6. Siraganian, R. P., and A. G. Osler. 1971. Destruction of rabbit platelets in the allergic response of sensitized leukocytes. I. Demonstration of a fluid phase intermediate. J. Immunol. 106: 1244-1251.

7. Kater, L. A., K. F. Austen, and E. J. Goetzl. 1975. Identification and partial purification of a platelet activating factor (PAF) from rat. Fed. Proc. 34: 985. (Abstr.)

8. Benveniste, J. 1974. Platelet-activating factor, a new mediator of anaphylaxis and immune complex deposition from rabbit and human basophils. Nature (Lond.). 249: 581-582.

9. Lewis, R. A., E. J. Goetzl, S. I. Wasserman, F. H. Valone, R. H. Rubin, and K. F. Austen. 1975. The release of four mediators of immediate hypersensitivity from human leukemia basophils. J. Immunol. 114: 87-92.

10. Benveniste, J. 1977. Platelet activating factor. Int. Arch. Allergy Appl. Immunol. In press.

11. Benveniste, J. 1974. Characteristics and semi purification of a platelet activating factor from human and rabbit leukocytes. Fed. Proc. 33: 797. (Abstr.)

12. Henson, P. M. 1976. Activation and desensitization of platelets by platelet-activating factor (PAF) derived from IgE-sensitized basophils. I. Characteristics of the secretory response. J. Exp. Med. 143: 937-952.

13. Henson, P. M., and Z. G. Oades. 1976. Activation of platelets by platelet-activating factor (PAF) derived from IgE-sensitized basophils. II. The role of serine proteases, cyclic nucleotides, and contractile elements in PAF-induced secretion. J. Exp. Med. 143: 953-968.

14. Henson, P. M., and R. N. Pinckard. 1977. Platelet activating factor (PAF). A direct mediator of anaphylaxis in the rabbit and a trigger for the vascular deposition of circulating immune complexes. In Mediators of the Immediate Type Inflammatory Reaction. K. Rother and A. deWeck, editors. S. Karger AG., Basel. In press.

15. Henson, P. M., and C. G. Cochrane. 1971. Immune complex disease in rabbits. The role of complement and of a leukocyte-dependent release of vasoactive amines from platelets. J. Exp. Med. 133: 554-571.

16. Kravis, T. C., and P. M. Henson. 1977. Accumulation of platelets at sites of antigen-antibody mediated injury. A possible role for IgE antibody and mast cells. J. Immunol. In press.

17. Halonen, M., R. N. Pinckard, and A. L. Meng. 1973. Characterization of IgE-induced systemic anaphylaxis in the rabbit. Lack of correlation between the intravascular release of histamine and anaphylactic hypersensitivity. J. Immunol. 111: 331-340.

18. Henson, P. M., and R. N. Pinckard. 1976. Platelet activating factor (PAF) as a mediator in IgE anaphylaxis. Fed. Proc. 35: 516. (Abstr.)

19. Holmsen, H. 1974. Are platelet shape change, aggregation and release reaction tangible manifestations of one basic platelet function? In Platelets, Production, Function, Transfusion, Storage. M. G. Baldini and S. Ebbe, editors. Grune \& Stratton, Inc., New York.

20. Haslam, R. J. 1964. Role of adenosine diphosphate in the aggregation of human blood platelets by thrombin and by fatty acids. Nature (Lond.). 202: 765-768. 
21. Haslam, R. J. 1967. Mechanisms of blood platelet aggregation. In Physiology of Hemostasis and Thrombosis. S. A. Johnson and W. H. Seegers, editors. Charles C. Thomas Publisher, Springfield, Ill. 88-112.

22. Izrael, V., K. Zawilska, F. Jaisson, S. Levy-Toledano, and J. Caen. 1974. In Platelets, Production, Function, Transfusion, Storage. M. G. Baldini and S. Ebbe, editors. Grune \& Stratton, Inc. New York. 187.

23. Packham, M. A., M. A. Guccione, P-L. Chang, and J. F. Mustard. 1973. Platelet aggregation and release: effects of low concentrations of thrombin or collagen. Am. J. Physiol. 225: 38-47.

24. Hamberg, M., Y. Svensson, and B. Samuelsson. 1975. Thromboxanes: a new group of biologically active compounds derived from prostaglandin endoperoxides. Proc. Natl. Acad. Sci. U. S. A. 72: 2994-2998.

25. Malmsten, C., M. Hamberg, J. Svensson, and B. Samuelsson. 1975. Physiologic role of an endoperoxide in human platelets: hemostatic defect due to platelet cyclo-oxygenase deficiency.Proc. Natl. Acad. Sci. U.S. A. 72: $1446-1450$.

26. Smith, J. B., C. Ingerman, J. J. Kocsis, and M. J. Silver. 1974. Formation of an intermediate in prostaglandin synthesis and its association with the platelet release reaction. J. Clin. Invest. 53: 1468-1472.

27. Aster, R. H., and J. H. Jandl. 1964. Platelet sequestration in man. J. Clin. Invest. 43: 843-855.

28. Henson, P. M. 1970. Mechanisms of release of constituents from rabbit platelets by antigen-antibody com- plexes and complement. I. Lytic and nonlytic reactions. J. Immunol. 105: 476-489.

29. Ardlie, N. G., M. A. Packham, and J. F. Mustard. 1970. Adenosine diphosphate-induced platelet aggregation in suspensions of washed rabbit platelets. $\mathrm{Br}$. J. Haematol. 19: 7-17.

30. Holmsen, H., E. Storm, and H. J. Day. 1972. Determination of ATP and ADP in blood platelets; a modification of the firefly luciferase assay for plasma. Anal. Biochem. 46: 489-501.

31. Stanley, P. E., and S. G. Williams. 1969. Use of the liquid scintillation spectrometer for determining adenosine triphosphate by the luciferase enzyme. Anal. Biochem. 29: 381-392.

32. Grette, K. 1962. Studies on the mechanism of thrombincatalyzed hemostatic reactions in blood platelets. Acta Physiol. Scand. 56(Suppl. 195): 5-93.

33. Henson, P. M., D. Gould, and E. L. Becker. 1976. Activation of stimulus-specific serine esterases (proteases) in the initiation of platelet secretion. I. Demonstration with organophosphorus inhibitors. J. Exp. Med. 144: $1657-73$.

34. Becker, E. L., and P. M. Henson. 1973. In vitro studies of immunologically induced secretion of mediators from cells and related phenomena. Adv. Immunol. 17: 93-178.

35. Shaw, J. O., M. Printz, R. Stewart, and P. Henson. 1977. Relationship of prostaglandin production to secretion in platelets stimualted with platelet activating factor (PAF). Fed. Proc. 36: 1329. (Abstr.) 